\title{
Analgesic effect of GT-0198, a structurally novel glycine transporter 2 inhibitor, on a mouse model of neuropathic pain
}

Yu Omori, Mayumi Nakajima, Kazumi Nishimura, Eiki Takahashi, Tadamasa Arai, Masato Akahira, Tomohiko Suzuki, Mie Kainoh

Pharmaceutical Research Laboratories, Toray Industries, Inc., Japan

This study was conducted to show the pharmacological characteristic features of GT-0198. GT-0198 inhibited the function of glycine transporter 2 (GlyT-2) in the human GlyT-2-expressed HEK293 cells and did not bind various major transporters and receptors of neurotransmitter in the competitive manner. Thus, GT-0198 was considered as a comparatively selective GlyT-2 inhibitor. Intravenous, oral and intrathecal injection of GT-0198 decreased the painrelated response on the model of neuropathic pain with the partial sciatic nerve ligation. This result suggests that GT -0198 has an analgesic effect. The analgesic effect of GT-0198 was abolished by the intrathecal injection of strychnine, a glycine receptor antagonist. Therefore, GT-0198 is considered to exhibit the analgesic effect via the activation of glycine receptor by glycine following the presynaptic GlyT-2 inhibition in the spinal cord. In summary, GT-0198 is a novel GlyT -2 inhibitor with in vivo efficacy in behavioral models of neuropathic pain. 